\title{
Hiding the high excitation in the head of a fast Herbig-Haro jet
}

\author{
A. C. Raga ${ }^{1}$ and J. Cantó ${ }^{2}$ \\ 1 Instituto de Ciencias Nucleares, UNAM, Ap. 70-543, 04510 D F, México \\ 2 Instituto de Astronomía, UNAM, Ap. 70-264, 04510 D F, México
}

Received 19 May 2003 / Accepted 4 September 2003

\begin{abstract}
We propose that some low excitation $\mathrm{HH}$ objects might correspond to the head of a pulsed jet moving into a dense environment. We develop an analytic model showing that the head of such a jet will move slowly, producing a low excitation bow shock. At the times at which the successive pulses catch up with the jet head, high excitation emission will be produced, lasting for a time of the order of the cooling timescale of the material heated in the pulse/jet head interaction. In this way, the jet head has "flashes" of high excitation emission superimposed on a "quiescent", low excitation emission.
\end{abstract}

Key words. ISM: Herbig-Haro objects - ISM: jets and outflows - ISM: kinematics and dynamics - shock waves

\section{Introduction}

The spectra of HH objects appear to fall in two more or less well defined categories: "low excitation spectra" (with strong lines of neutrals and some singly ionised species) and "high excitation spectra" (with strong lines of singly and twice ionised species). An attempt to quantify these two spectral categories can be found in Raga et al. (1996).

A well studied (but not fully understood) effect is that the knots along a $\mathrm{HH}$ jet have low excitation spectra implying shock velocities of $\sim 10 \rightarrow 70 \mathrm{~km} \mathrm{~s}^{-1}$, which are considerably lower than the $\sim 200 \rightarrow 300 \mathrm{~km} \mathrm{~s}^{-1}$ measured jet velocities. Three different possibilities have been proposed in order to explain this effect:

- that the emission is produced in highly oblique, stationary shocks;

- that the emission comes from oblique, travelling shocks;

- that the emission is produced in 2-shock "internal working surfaces" travelling down the jet beam.

The first of these possibilities was studied in some detail by Falle et al. (1987), Cantó et al. (1989) and Raga et al. (1991), but has been mostly elliminated by the discovery of high proper motions in the knots along the HH 34 and HH 111 jets (Heathcote \& Reipurth 1992; Eislöffel \& Mundt 1992; Reipurth et al. 1992). The second idea was explored in detail by Micono et al. (1998), who showed that this kind of model can explain both the line ratios and the proper motions of the knots along HH jets. The third model has been studied in even more detail (Raga et al. 1990; Stone \& Norman 1993; de Gouveia Dal Pino \& Benz 1994; Völker et al. 1999; Cantó et al. 2000), and has been used for attempting to reproduce in a

Send offprint requests to: A. Raga, e-mail: raga@nuclecu. unam.mx detailed way the observational properties of HH 34 and HH 111 (Raga \& Noriega-Crespo 1998; Masciadri et al. 2002,b; Raga et al. 2002).

It is of course less clear how to obtain low excitation emission in the leading head of an HH jet. In the head of a jet, the jet velocity is split between the bow shock and the Mach disk (or "jet shock"). Therefore the head of a $300 \mathrm{~km} \mathrm{~s}^{-1}$ jet will have one shock with a shock velocity $v_{s 1} \leq 150 \mathrm{~km} \mathrm{~s}^{-1}$ and a second shock with $v_{s 2} \geq 150 \mathrm{~km} \mathrm{~s}^{-1}$ (whether the bow shock or the Mach disk is the faster shock being decided by the jet-to-ambient density ratio, see Hartigan 1989; Raga \& Noriega-Crespo 1993) and should therefore have a high excitation spectrum.

A possibility for explaining an observed low excitation "head" is to have a significant velocity decrease along the beam of the jet (which could be the result of a "slow startup" of the ejection or more likely a result of the interaction with the surrounding environment). In this way one has a fast flow close to the source (as observed) and a slower flow immediately upstream of the head of the jet. This possibility has been explored in the context of the HH 34 giant jet by Cabrit \& Raga (2000), de Gouveia Dal Pino (2001) and Masciadri et al. (2002). It is not clear, however, whether such models could explain the low excitation heads of "shorter" flows such as HH 7-11 (see, e.g., Noriega-Crespo \& Garnavich 2001).

In the present paper we propose the following, alternative model. A jet with a variable ejection velocity generates a series of "internal working surfaces" which contain most of the material of the outflow (see, Raga \& Kofman 1992; Cantó et al. 2000). These internal working surfaces move at a velocity $v_{0}$ which is determined by the mass and momentum contained in each "ejection event" or "pulse" of the time-dependent ejection (this velocity does not depend on the properties of the 


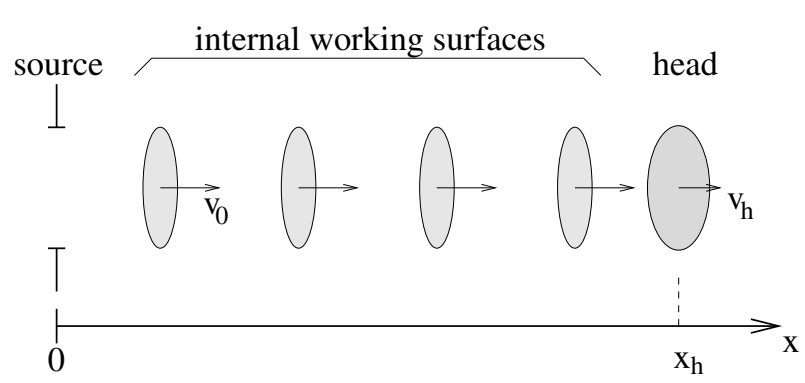

Fig. 1. Schematic diagram showing the structure of a pulsed $\mathrm{HH}$ jet. The "internal working surfaces" are idealized as clumps travelling at a (constant) velocity $v_{0}$. The head of the jet (at position $x_{\mathrm{h}}$ ) travels at a velocity $v_{\mathrm{h}}<v_{0}$. The $x$-coordinate is measured from the source, along the outflow axis.

surrounding environment, as the working surfaces travel into the "channel" which is left behind by the passage of the head of the jet). If the jet travels into a dense enough environment, the head of the jet will move at a low velocity, and will be impacted upon by the successive "clumps" (i.e., the internal working surfaces) which catch up with the jet head.

In this flow configuration, the head of the jet has a low velocity bow shock, and therefore produces a low excitation spectrum. As the jet beam is composed of "clumps" (rather than being a continuous flow), the head has no Mach disk, except at the times at which the clumps impact on the head. Therefore, the high excitation spectrum of the Mach disk is absent at most times. In this way, a variable jet moving into a high density environment will produce a slow moving head, which most of the time emits a low excitation spectrum, and emits a high excitation spectrum only in "flashes" corresponding to the times in which the successive internal working surfaces catch up with the jet head.

In Sect. 2, we present a simple, analytic model (based on mass and momentum conservation considerations) describing the motion of the head of the jet. The emission properties of the jet head are described in Sect. 3. Finally, the results, implications and limitations of the model are discussed in Sect. 4.

\section{The motion of the head of a variable jet}

In order to derive the equation of motion for the head of a variable jet we consider the situation shown in the schematic diagram of Fig. 1. We assume that the jet beam is formed by a series of "clumps", which travel away from the source at a velocity $v_{0}$. These clumps could be directly associated with the form of the time-dependent ejection, or alternatively could be the result of an abritrary ejection velocity variability which then leads to the formation of internal working surfaces which contain most of the mass and momentum of the jet beam (see Raga \& Kofman 1992; Cantó et al. 2000).

Due to its direct interaction with the environment, the head of the jet moves with a velocity $v_{\mathrm{h}}<v_{0}$. Therefore the "clumps" along the jet beam catch up with the head, supplying it with more mass and momentum.

Figure 2 shows the situation found for a periodic ejection variability with pulses of mass $M_{0}$ and velocity $v_{0}$ ejected with a period $\tau$. The first "clump" is ejected at $t_{1}=0$, and

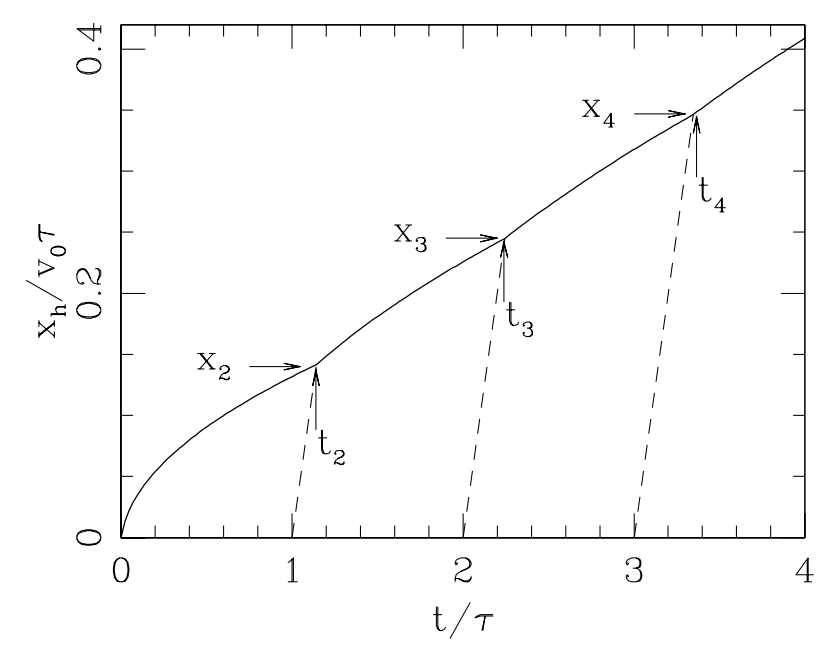

Fig. 2. Diagram showing the position $x_{\mathrm{h}}$ of the head of the jet as a function of time. The solid line shows $x_{\mathrm{h}}(t)$, and the dashed lines represent the motion of the successive pulses, which leave the source at times $\tau, 2 \tau, 3 \tau, \ldots$ and catch up with the jet head at times $t_{2}, t_{3}, t_{4}, \ldots$ and at positions $x_{2}, x_{3}, x_{4}, \ldots$, respectively.

starts interacting with the environment at $x_{1}=0$ (where the $x$-coordinate corresponds to the distance from the source along the outflow axis, see Fig. 1).

The following clumps are ejected from the source at times $t=\tau, 2 \tau, 3 \tau$, etc., and are assumed to travel unimpeded at a velocity $v_{0}$ until they catch up with the head of the jet. We assume that these clumps do not expand sideways beyond the "channel" left behind by the jet head, which is probably reasonable given the very high Mach numbers (of $\sim 10 \rightarrow 100$ ) estimated for $\mathrm{HH}$ jets. The trajectories of these clumps in the $(x, t)$ plane are depicted by the dashed lines in Fig. 2. The successive clumps catch up with the jet head at positions and times $\left(x_{2}, t_{2}\right),\left(x_{3}, t_{3}\right)$, etc. (see Fig. 2).

Depending on the parameters of the flow, one could have situations in which the successive clumps do not travel into an "empty channel" and could be dynamically affected by their interaction with the material filling up the interclump region. For this to take place, the gas in the "cocoon" surrounding the jet beam (which is composed of shocked environmental material and jet material ejected sideways by the jet head) has to be able to fill in the channel in the period $\tau$ between the passage of two successive knots. The condition for the channel to be filled in is therefore

$\tau>300 \mathrm{yr}\left(\frac{r_{j}}{10^{16} \mathrm{~cm}}\right)\left(\frac{10 \mathrm{~km} \mathrm{~s}^{-1}}{c_{0}}\right)$,

where $c_{0}$ is the sound speed of the material in the cocoon. Therefore, the assumption that the successive clumps travel at a constant velocity $v_{0}$ is valid only for sufficiently short source variability periods (see Eq. (1)).

Let us now derive the equation of motion for the position $x_{\mathrm{h}}$ of the head of the jet (see Fig. 2) for the segment of its trajectory between the points $\left(x_{n}, t_{n}\right)$ and $\left(x_{n+1}, t_{n+1}\right)$. If we assume that the jet has a position-independent radius $r_{j}$, and that the head of the jet has all of the mass of the "clumps" that have reached it as 
well as the mass of the swept-up environment, the mass of the head is then given by:

$M_{\mathrm{h}}=n M_{0}+\pi r_{j}^{2} \rho_{\mathrm{env}} x_{\mathrm{h}}$,

where $\rho_{\mathrm{env}}$ is the density of the uniform environment. The momentum of the jet head is

$\Pi_{\mathrm{h}}=n M_{0} v_{0}$,

where $v_{0}$ is the velocity of the clumps. The equation of motion then is

$v_{\mathrm{h}}=\frac{\mathrm{d} x_{\mathrm{h}}}{\mathrm{d} t}=\frac{\Pi_{\mathrm{h}}}{M_{\mathrm{h}}}=\frac{n M_{0} v_{0}}{n M_{0}+\pi r_{j}^{2} \rho_{\mathrm{env}} x_{\mathrm{h}}}$,

which can be integrated in a straightforward way to obtain

$\left(\frac{x_{\mathrm{h}}-x_{n}}{v_{0} \tau}\right)+\frac{1}{2 n \beta}\left(\frac{x_{\mathrm{h}}^{2}-x_{n}^{2}}{v_{0}^{2} \tau^{2}}\right)=\left(\frac{t-t_{n}}{\tau}\right)$,

where $\beta=\overline{\rho_{j}} / \rho_{\text {env }}$, with the average jet density $\overline{\rho_{j}}$ being defined as:

$\overline{\rho_{j}}=\frac{M_{0}}{\pi r_{j}^{2} v_{0} \tau}$

The segment of the $x_{\mathrm{h}}$ vs. $t$ relation given by Eq. (5) ends at a position/time $\left(x_{n+1}, t_{n+1}\right)$, at which the $(n+1)$ th pulse catches up with the jet head (see Fig. 2). The $(n+1)$ th pulse follows a trajectory

$x^{(n+1)}=(t-n \tau) v_{0}$

and we can then obtain $\left(x_{n+1}, t_{n+1}\right)$ by finding the point at which the trajectory of the pulse (Eq. (7)) intersects the trajectory of the jet head (Eq. (5)). Combining Eqs. (5) and (7) one obtains the recurrence relation

$$
\begin{aligned}
\left(\frac{t_{n+1}}{\tau}\right)^{2} & -\left(\frac{t_{n}}{\tau}\right)^{2}-2 n\left(\frac{t_{n+1}}{\tau}\right) \\
& +2(n-1)\left(\frac{t_{n}}{\tau}\right)+2 n(1-\beta)-1=0,
\end{aligned}
$$

relating $t_{n}$ and $t_{n+1}$. It is straightforward to show that the solution to this recurrence relation is

$\left(\frac{t_{n}}{\tau}\right)=n-1+\sqrt{n(n-1) \beta}$.

Substituting Eq. (9) into (7), we obtain

$\left(\frac{x_{n}}{v_{0} \tau}\right)=\sqrt{n(n-1) \beta}$.

Equations (9) and (10) then give the time and position at which the $n$th pulse catches up with the jet head. These equations can be combined with Eq. (5) to give the trajectory

$\left(\frac{x_{\mathrm{h}}}{v_{0} \tau}\right)=-n \beta+\sqrt{n \beta[n \beta-n+1+2(t / \tau)]}$

followed by the jet head for times $t_{n} \leq t \leq t_{n+1}$. Putting $n=1,2,3, \ldots$ we then obtain the successive uniformly continuous segments of the trajectory of the jet head shown in Fig. 2.

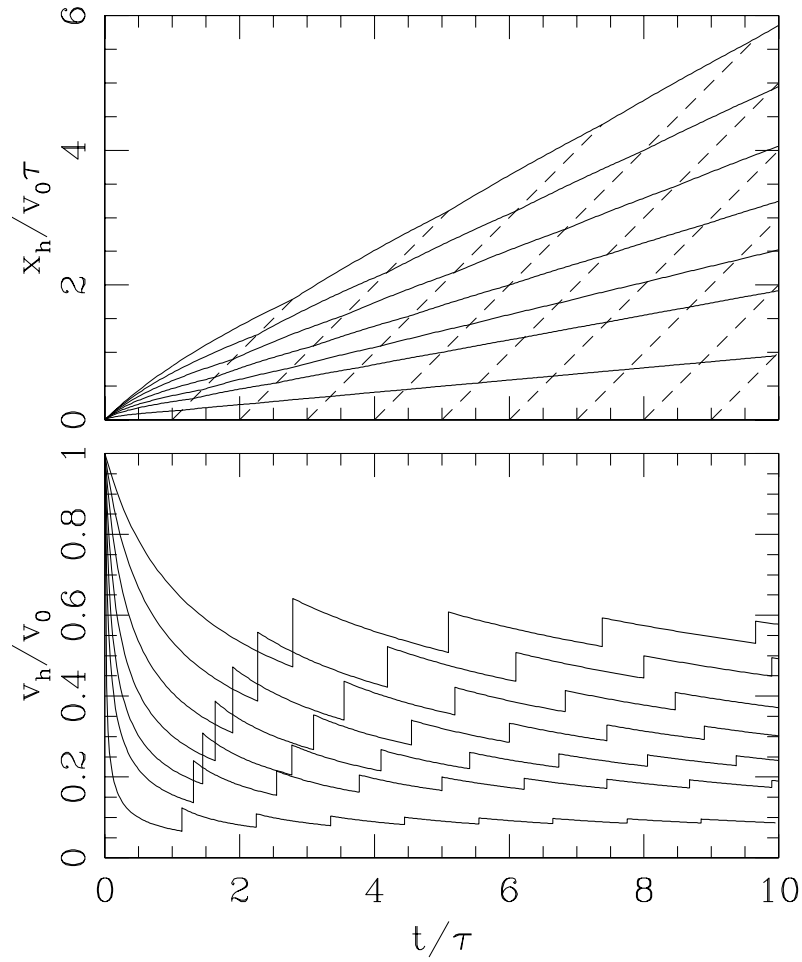

Fig. 3. Solutions for the position $x_{\mathrm{h}}(t)$ (top) and the velocity $v_{\mathrm{h}}(t)$ (bottom) of the head of the jet for models with $\beta=0.01$ (lower curve), $0.05,0.1,0.2,0.4,0.8$ and 1.6 (upper curve in both graphs). The dashed lines (top) represent the motion of the successive pulses, and the crossing points between these lines and the solid, $x_{\mathrm{h}}(t)$ curves correspond to the points at which the successive pulses catch up with the jet head. At these points, the velocity $v_{\mathrm{h}}(t)$ of the jet head shows discontinuous increases (bottom graph).

The time-dependent velocity $v_{\mathrm{h}}$ of the jet head can then be computed from Eq. (4) as

$\left(\frac{v_{\mathrm{h}}}{v_{0}}\right)=\left[1+\frac{1}{n \beta}\left(\frac{x_{\mathrm{h}}}{v_{0} \tau}\right)\right]^{-1}$.

In Fig. 3, we show the solution for the motion of the jet head obtained from Eqs. (11) and (12) for different values of the dimensionless parameter $\beta=\overline{\rho_{j}} / \rho_{\text {env }}$ (see Eq. (6)). From this figure, it is clear that the velocity of the jet head has higher values for the solutions with larger $\beta$.

We can also see from Fig. 3 that while the position $x_{\mathrm{h}}$ of the jet head is (as hoped) continuous as a function of time, its velocity $v_{\mathrm{h}}$ is not, as it has discontinuous jumps at the times at which the clumps catch up with the jet head.

By combining Eqs. (10) and (12) we can obtain the velocities immediately to the left

$\left(\frac{v_{n, l}}{v_{0}}\right)=\left[1+\sqrt{\frac{n}{(n-1) \beta}}\right]^{-1}$,

and to the right

$\left(\frac{v_{n, r}}{v_{0}}\right)=\left[1+\sqrt{\frac{(n-1)}{n \beta}}\right]^{-1}$, 
of the $(n-1)$ th discontinuity of the velocity vs. time curves shown in the bottom panel of Fig. 3. It is clear that for large $n$ both $v_{n, l}$ and $v_{n, r}$ asymptotically reach the value

$\left(\frac{v_{\infty}}{v_{0}}\right)=\left[1+\beta^{-1 / 2}\right]^{-1}$,

which (as is evident to the connaisseur) corresponds to the motion resulting from the ram pressure balance of a jet with a time-independent density $\overline{\rho_{j}}$ and velocity $v_{0}$ moving into an environment of density $\rho_{\text {env }}$. As the velocity $v_{\mathrm{h}}$ in the $n$th continuous segment always satisfies the condition $v_{n, r} \geq v_{\mathrm{h}} \geq v_{n+1, l}$, for large values of $n$ the velocity as a function of time also converges to the constant value $v_{\infty}$ given by Eq. (15).

\section{The emission properties}

\subsection{Quiescent emission}

The continuous segments of the $v_{\mathrm{h}}$ vs. $t$ dependence of the motion of the head of the jet (see Fig. 3) emit energy in a continuous way as a function of time. Considering that for a high Mach number, highly radiative flow the thermal energy is negligible, then the rate of change of the kinetic energy of the head of the jet (in the continuous segments, in which no new kinetic energy is being injected into the head) has to be equal to the radiative luminosity of the flow:

$$
L_{\mathrm{c}}=-\frac{\mathrm{d} E_{\mathrm{kin}}}{\mathrm{d} t}=\frac{\pi r_{j}^{2} \rho_{\mathrm{env}} v_{0}^{3}}{2\left[1+\left(\frac{x}{v_{0} \tau}\right) \frac{1}{n \beta}\right]^{3}} \rightarrow \frac{\pi r_{j}^{2} \rho_{\mathrm{e}} v_{0}^{3}}{2\left(1+\beta^{-1 / 2}\right)},
$$

where we have used the fact that $E_{\text {kin }}=M_{\mathrm{h}} v_{\mathrm{h}}{ }^{2} / 2$ and Eqs. (2) and (12). The second expression of Eq. (16) corresponds to the $n \gg 1$ limit, which can be obtained in an analogous way to the one described for deriving Eq. (15).

\subsection{Flashes}

When the successive clumps catch up with the head of the jet, we have a "discrete" amount of energy which is emitted as a result of the interaction process. This energy can be computed in a straightforward way as the difference between the clump+head kinetic energy (just before the interaction), and the kinetic energy of the "merged" clump+head:

$$
\begin{aligned}
\Delta E_{k}^{(n)}= & \frac{1}{2} M_{0} v_{0}^{2}+\frac{1}{2}\left[(n-1) M_{0}+\pi r_{j}^{2} \rho_{\mathrm{env}} x_{n}\right] v_{n, l}{ }^{2} \\
& -\frac{1}{2}\left[n M_{0}+\pi r_{j}^{2} \rho_{\mathrm{env}} x_{n}\right] v_{n, r}{ }^{2} .
\end{aligned}
$$

Using Eqs. (13) and (14) we then obtain

$$
\begin{aligned}
\Delta E_{k}^{(n)} & =\frac{\pi r_{j}^{2} \rho_{\mathrm{env}} v_{0}^{3} \beta \tau}{2\left[1+\sqrt{\frac{n \beta}{n-1}}\right]\left[1+\sqrt{\frac{(n-1) \beta}{n}}\right]} \\
& \rightarrow \frac{\pi r_{j}^{2} \rho_{\mathrm{env}} v_{0}^{3} \tau}{2\left(1+\beta^{-1 / 2}\right)^{2}},
\end{aligned}
$$

where the second relation corresponds to the $n \gg 1$ limit.

Of course, this energy is not ejected at a single instant (as implied by our simplified analytic model), but extends over a

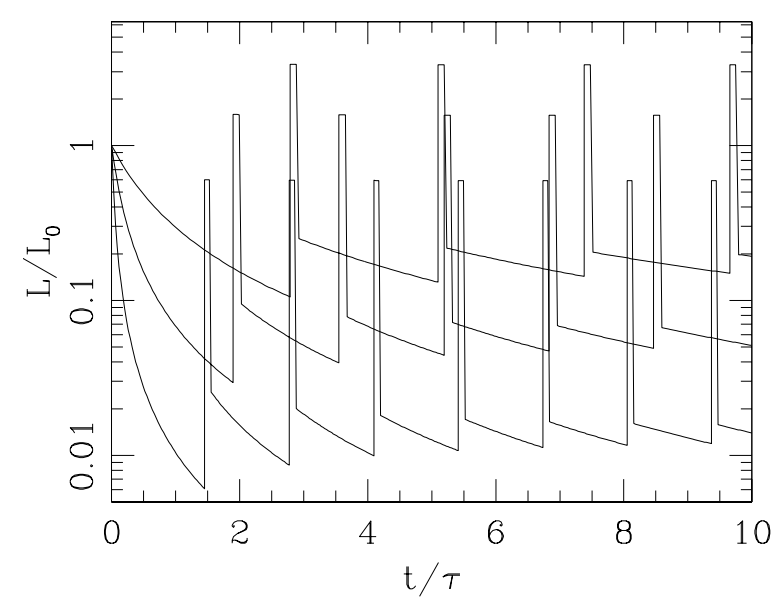

Fig. 4. Total luminosity of the jet head (in units of $L_{0}=\pi r_{j}^{2} \rho_{\text {env }} v_{0}^{3} / 2$ ) as a function of time for models with $\beta=0.1$ (lower curve), 0.4 and 1.6 (upper curve). We have assumed that the energy emitted in the "flashes" is distributed as a uniform luminosity over a time $\tau_{\mathrm{f}}=0.1 \tau$ immediately following each catching up process (in other words, we have set $\alpha=0.1$, see the text).

time $\tau_{\mathrm{f}}$ which depends on the size of the clumps along the symmetry axis (which has been implicitly set to zero in Sect. 2) and on the cooling timescale of the gas heated in the collision of the clump with the jet head.

If the emission due to the clump/head collision "flashes" lasts for a time $\tau_{\mathrm{f}}=\alpha \tau$, the resulting luminositiy would be given by

$L_{\mathrm{f}}^{(n)}=\frac{\pi r_{j}^{2} \rho_{\mathrm{env}} v_{0}^{3} \beta}{2 \alpha\left[1+\sqrt{\frac{n \beta}{n-1}}\right]\left[1+\sqrt{\frac{(n-1) \beta}{n}}\right]}$,

and the emission would last from time $t_{n}$ to $t_{n}+\alpha \tau$.

\subsection{Time-history of the emission}

In Fig. 4, we show the total luminosity $L=L_{\mathrm{c}}+L_{\mathrm{f}}$ (see Eqs. (16) and (19)) as a function of time for a model with $\alpha=\tau_{\mathrm{f}} / \tau=0.1$ and different values of $\beta$. For this value of $\alpha$, the "flashes" (resulting from the clump/jet head catching up processes) last for $10 \%$ of the time, and have luminosities which are 1.5 to 2 orders of magnitude higher than the luminosities of the "quiescent" periods between the flashes.

In the "small $\alpha$ " regime (i.e., in which the interaction+cooling time $\tau_{\mathrm{f}}$ is shorter than the period of the ejection variability), we then have short "flashes" separated by long quiescent periods. In models with low $\beta=\overline{\rho_{j}} / \rho_{\mathrm{e}}$, the velocity $v_{\mathrm{h}}$ of the jet head will be substantially lower than the jet velocity $v_{0}$ (see Eqs. (12) and (15)). Therefore, in the quiescent periods (when the emission comes from the bow shock which has a shock velocity equal to $v_{\mathrm{h}}$ ) will have a low excitation spectrum.

In the "flashes", we will see the emission of a shock of velocity $v_{0}-v_{\mathrm{h}}$ (which is comparable to $v_{0}$ in the $\beta \ll 1$ regime) lasting for the duration time of the clump/jet head collision. As the clumps along $\mathrm{HH}$ jets do not show large extensions along the outflow axis, the interaction probably lasts only for a time which is short in comparison to the ejection variability 
period. After the clump has completely merged with the jet head, the material heated in the interaction will cool radiatively, first emitting a high excitation spectrum, later emitting a lower excitation spectrum, and finally fading away.

Interestingly, the cooling timescale depends on the inverse of the density, and is strongly dependent on the shock velocity. For the $n_{j} \sim 10^{2} \rightarrow 10^{4} \mathrm{~cm}^{-3}$ (number) density range and $v_{0} \sim 100 \rightarrow 300 \mathrm{~km} \mathrm{~s}^{-1}$ velocity range relevant for $\mathrm{HH}$ jets, the cooling timescale ranges from about one month up to $\tau_{\mathrm{c}} \sim 200 \mathrm{yr}$ (see, e.g., Hartigan et al. 1987). Since, the ejection variability periods that have been estimated for $\mathrm{HH}$ jets lie in the $\tau \sim 30 \rightarrow 1500 \mathrm{yr}$ range (Raga et al. 2002), it is clear that models with a wide range of $\alpha=\tau_{\mathrm{f}} / \tau$ might be relevant for different $\mathrm{HH}$ jets.

\section{Conclusions}

We have presented a simple model (based on considerations of mass and momentum conservation) for the motion of the head of a variable jet. The ejection variability leads to the formation of discrete "clumps" along the body of the jet, and these clumps catch up with the jet head, supplying it with mass and momentum from the jet beam.

Interestingly, for the case of a perfectly collimated jet (i.e., with zero opening angle) moving into a uniform environment this model leads to a full analytic solution, which illustrates the properties of this kind of flow. The analytic solution shows that in the early evolution of the flow, the head of the jet first advances with the velocity $v_{0}$ of the clumps, and then slows down (as it incorporates environmental material) monotonically until it is caught up by the second clump. The increased momentum gives the head a larger velocity, which then decreases again as the head travels away from the source (see Fig. 3). After the head has been caught up by $\sim 5$ clumps, it has enough inertia that it coasts along at a more or less constant velocity, which is a function of the time-averaged properties of the jet and of the environmental density (see Fig. 3 and Eq. (15)).

If one has a jet with a low value of $\beta=\overline{\rho_{j}} / \rho_{\text {env }}$ (see Eq. (6)), the jet head travels at a low velocity (see Eq. (15)), and therefore has a low excitation bow shock. At the times at which the clumps catch up with the jet head, the emitted spectrum has "flashes" of emission which result from the dissipation in the clump/jet head interaction. For low $\beta$, the shock velocities associated with this interaction will have a shock velocity $\sim v_{0}$, therefore having a high excitation spectrum.

These "flashes" will last for the sum of the (short) interaction timescale and the cooling timescale of the shocked material. As discussed in Sect. 3.3, for the parameters of $\mathrm{HH}$ jets it is definitely possible to have cooling timescales $\tau_{\mathrm{c}} \ll \tau$ (where $\tau$ is the ejection variability period). For such parameters, the emission of the jet head will have periods of "quiescent", low excitation emission separated by short-lived, bright "flashes" of high excitation emission.
This regime for the motion of the head of a variable jet is clearly interesting from the point of view of modelling low excitation $\mathrm{HH}$ objects. In the future, this scenario should be tested by attempting to model individual low excitation $\mathrm{HH}$ objects with full gasdynamical numerical simulations.

Another interesting possible application of our model is the observation of non-thermal radio emission associated with outflows from young stars (see, e.g., Rodríguez et al. 1989). Henriksen et al. (1991) have shown that this non-thermal emission could be associated with particle acceleration in fast shocks in $\mathrm{HH}$ jets. The present model, in which fast shocks are present only for short time-periods could reconcile the existence of non-thermal emission in regions in which a high excitation emission line spectrum is currently not observed.

Acknowledgements. We would like to acknowledge support from the CONACyT grants 36572-E and 41320 and the DGAPA (UNAM) grant IN 112602.

\section{References}

Cabrit, S., \& Raga, A. C. 2000, A\&A, 354, 667

Cantó, J., Raga, A. C., \& Binette, L. 1989, RMxAA, 17, 65

Cantó, J., Raga, A. C., \& D’Alessio, P. 2000, MNRAS, 313, 656

Eislöffel, J., \& Mundt, R. 1992, A\&A, 263, 292

Falle, S. A. E. G., Innes, D. E., \& Wilson, M. J. 1987, MNRAS, 225, 741

de Gouveia Dal Pino, E. M. 2001, ApJ, 551, 347

de Gouveia Dal Pino, E. M., \& Benz, W. 1994, ApJ, 435, 261

Hartigan, P. 1989, ApJ, 339, 987

Hartigan, P., Raymond, J. C., \& Hartmann, L. W. 1987, ApJ, 323, 348

Heathcote, S., \& Reipurth, B. 1992, AJ, 104, 2193

Henriksen, R. N., Ptuskin, V. S., \& Mirabel, L. F. 1991, A\&A, 248, 221

Masciadri, E., Velázquez, P. F., Raga, A. C., Cantó, J., \& Noriega-Crespo, A. 2002a, ApJ, 573, 260

Masciadri, E., de Gouveia Dal Pino, E. M., Raga, A. C., \& Noriega-Crespo, A. 2002b, ApJ, 580, 950

Micono, M., Massaglia, S., Bodo, G., Rossi, P., \& Ferrari, A. 1998, A\&A, 333, 1001

Noriega-Crespo, A., \& Garnavich, P. M. 2001, AJ, 122, 3317

Raga, A. C., Cantó, J., Binette, L., \& Calvet, N. 1990, ApJ, 364, 601

Raga, A. C., Biro, S., Cantó, J., \& Binette, L. 1991, RMxAA, 22, 243

Raga, A. C., Böhm, K. H., \& Cantó, J. 1996, RMxAA, 32, 161

Raga, A. C., \& Noriega-Crespo, A. 1998, AJ, 116, 2943

Raga, A. C., \& Noriega-Crespo, A. 1993, RMxAA, 25, 149

Raga, A. C., \& Kofman, L. 1992, ApJ, 390, 359

Raga, A. C., Velázquez, P. F., Cantó, J., \& Masciadri, E. 2002, A\&A, 395, 647

Reipurth, B., Raga, A. C., \& Heathcote, S. 1992, ApJ, 392, 145

Rodríguez, L. F., Curiel, S., Moran, J. M., et al. 1989, ApJ, 346, L85

Stone, J. M., \& Norman, M. L. 1993, ApJ, 413, 198

Völker, R., Smith, M. D., Suttner, G., \& Yorke, H. W. 1999, A\&A, 343, 953 